\title{
NGC 4654: Gravitational interaction or ram pressure stripping?
}

\author{
B. Vollmer \\ Max-Planck-Institut für Radioastronomie, Auf dem Hügel 69, 53121 Bonn, Germany \\ Received 13 July 2001 / Accepted 5 November 2002

\begin{abstract}
The Virgo cluster spiral galaxy NGC 4654 is supposed to be a good candidate for ongoing ram pressure stripping based on its very asymmetric Hi distribution. However, this galaxy also shows an asymmetric stellar distribution. Numerical simulations using ram pressure as the only perturbation can produce a tail structure of the gas content, but cannot account for its kinematical structure. It is shown that a strong edge-on stripping event can produce an asymmetric stellar distribution up to $800 \mathrm{Myr}$ after the stripping event, i.e. the galaxy's closest passage to the cluster center. Simulations using a gravitational interaction with the companion galaxy NGC 4639 can account for the asymmetric stellar distribution of NGC 4654, but cannot properties of NGC 4654. It is concluded that NGC 4654 had a tidal interaction $~ 500 \mathrm{Myr}$ ago and is continuing to experience ram pressure until the present day.
\end{abstract} \\ reproduce the observed extended gas tail. Only a mixed interaction, gravitational and ram pressure, can reproduce all observed
}

Key words. galaxies: individual: NGC 4654 - galaxies: interactions - galaxies: ISM - galaxies: kinematics and dynamics

\section{Introduction}

Maps of the gas content of spiral galaxies in the Virgo cluster have revealed that the Hi disks of cluster spirals are disturbed and considerably reduced (Cayatte et al. 1990, 1994). Despite their Hi deficiency, these spiral galaxies do not show a lack of molecular gas (Kenney \& Young 1989). These observational results suggest that gas removal due to the rapid motion of the galaxy within the intracluster medium (ICM) (rampressure stripping; Gunn \& Gott 1972) is responsible for the $\mathrm{HI}$ deficiency and the disturbed gas disks of theses cluster spirals. Nevertheless, it is still an open question where and when these galaxies lost their atomic gas.

In order to study in detail how ram pressure acts, we are modeling galaxy orbits and use a three-dimensional $\mathrm{N}$-body code to simulate the gas kinematics of a spiral galaxy falling into the Virgo cluster (Vollmer et al. 2001). Ram pressure exerted by the ICM on the ISM of a rapidly moving galaxy is explicitly included in this code.

One candidate for an ISM-ICM interaction in the Virgo cluster is NGC 4654. Its physical parameters are listed in Table 1. Phookun \& Mundy (1995) observed this galaxy in the 21-cm-line with a high sensitivity $\left(\sim 10^{19} \mathrm{~cm}^{-2}\right)$ at the VLA. They found a very asymmetric Hi distribution with a compressed edge on one side and a long tenuous tail on the other side. They argued that this asymmetry is due to ram pressure stripping that pushes the ISM beyond the galaxy's optical radius.

\footnotetext{
* e-mail: bvollmer@mpifr-bonn.mpg.de
}

Table 1. Physical parameters of NGC 4654.

\begin{tabular}{|c|c|}
\hline Other names & $\begin{array}{r}\text { UGC } 7902 \\
\text { VCC } 1987 \\
\text { CGCG } 071-019\end{array}$ \\
\hline$\alpha(2000)^{\mathrm{a}}$ & $12^{\mathrm{h}} 43^{\mathrm{m}} 56.6^{\mathrm{s}}$ \\
\hline$\delta(2000)^{\mathrm{a}}$ & $13^{\circ} 07^{\prime} 36^{\prime \prime}$ \\
\hline Morphological type $^{\mathrm{a}}$ & SBcd \\
\hline Distance to the cluster center $\left({ }^{\circ}\right)$ & 3.4 \\
\hline Optical diameter $D_{25}{ }^{\text {a }}\left(^{\prime}\right)$ & 4.9 \\
\hline$B_{T}^{0 \mathrm{a}}$ & 10.75 \\
\hline Systemic heliocentric velocity ${ }^{\mathrm{a}}\left(\mathrm{km} \mathrm{s}^{-1}\right)$ & $1035 \pm 3$ \\
\hline Distance $D(\mathrm{Mpc})$ & 17 \\
\hline$V_{\text {rot }_{\max }}\left(\mathrm{km} \mathrm{s}^{-1}\right)$ & $130^{\mathrm{b}}, 139^{\mathrm{c}}$ \\
\hline PA & $121^{\mathrm{ob}}, 128^{\mathrm{o}} \mathrm{c}$ \\
\hline Inclination angle & $49^{\circ \mathrm{b}}, 51^{\mathrm{oc}}$ \\
\hline HI deficiency ${ }^{\mathrm{d}}$ & $0.17 \pm 0.2$ \\
\hline
\end{tabular}

a RC3, de Vaucouleurs et al. (1991).

b Guharthakurta et al. (1988).

c Sperandio et al. (1995).

d Cayatte et al. (1994).

However, NGC 4654 does not only show an asymmetric gas disk, but also has an asymmetric stellar distribution. This might be due to a gravitational interaction with the nearby spiral galaxy NGC 4639.

In order to investigate the possibilities of tidal or ISM-ISM interaction, the code presented in Vollmer et al. (2001) has been extended and contains now a non-collisional component. This 
gives the opportunity to make simulations of a gravitational interaction with or without additional ram pressure.

In this article I compare snapshots of four different simulations with Hi observations of NGC 4654:

1. ram pressure, analytically given gravitational potential of the galaxy;

2. ram pressure, galactic halo, bulge, and disk simulated by non-collisional particles;

3. gravitational interaction;

4. gravitational interaction and ram pressure.

The comparison of the gas distribution and velocity field permits to discriminate between the different scenarios.

The plan of this article is the following: the numerical code is described in Sect. 2. The simulations using ram pressure as the only perturbation and their comparison with observations are presented in Sect. 4. In Sect. 5 a scenario of active stripping is discussed. The simulations using a gravitational interaction with and without ram pressure and their comparison with observations are presented in Sect. 6, followed by a discussion of the results of all simulations in Sect. 7. A summary and conclusions are given in Sect. 8 .

\section{The model}

\subsection{The collisional component}

Since the model is described in detail in Vollmer et al. (2001), I will only briefly summarize its main features. The particles represent gas cloud complexes which are evolving in an analytically given gravitational potential of the galaxy.

10000 particles of different masses are rotating within this gravitational potential. The total gas mass is $M_{\text {gas }}^{\text {tot }}=5.8 \times$ $10^{9} M_{\odot}$, which is $\sim 30 \%$ larger than the observed gas mass (Huchtmeier \& Richter 1989). To each particle a radius is attributed depending on its mass. During the disk evolution the particles can have inelastic collisions, the outcome of which (coalescence, mass exchange, or fragmentation) is simplified following Wiegel (1994). This results in an effective gas viscosity in the disk.

As the galaxy moves through the ICM, its clouds are accelerated by ram pressure. Within the galaxy's inertial system its clouds are exposed to a wind coming from the opposite direction of the galaxy's motion through the ICM. The temporal ram pressure profile has the form of a Lorentzian, which is realistic for galaxies on highly eccentric orbits within the Virgo cluster (Vollmer et al. 2001). The effect of ram pressure on the clouds is simulated by an additional force on the clouds in the wind direction. Only clouds which are not protected against the wind by other clouds are affected.

\subsection{The analytical gravitational potential}

The fixed, analytical gravitational potential consists of three parts: the dark matter halo, the stellar bulge, and the disk potential (Allen \& Santillán 1991). Using their definitions, the model parameters are: (i) halo: $a_{3}=12 \mathrm{kpc}$ (effective radius), $M_{3}=8.6 \times 10^{10} M_{\odot}$ (halo mass), (ii) bulge: $b_{1}=390 \mathrm{pc}$
Table 2. Number of particles $N$, particle mass $M$, and smoothing length $l$ for the different galactic components.

\begin{tabular}{llll}
\hline \hline component & $N$ & $M\left(M_{\odot}\right)$ & $l(\mathrm{pc})$ \\
\hline halo & 16384 & $9.2 \times 10^{6}$ & 1200 \\
bulge & 16384 & $3.2 \times 10^{5}$ & 180 \\
disk & 16384 & $1.6 \times 10^{6}$ & 240 \\
companion & 11000 & $9.2 \times 10^{6}$ & 1200 \\
\hline
\end{tabular}

(bulge core radius), $M_{1}=5.6 \times 10^{9} M_{\odot}$ (bulge mass), (iii) disk: $a_{2}=2.7 \mathrm{kpc}$ (disk scale length), $b_{2}=250 \mathrm{pc}$ (disk scale height), $M_{2}=2.6 \times 10^{10} M_{\odot}$ (mass of the stellar disk). The resulting velocity field has a constant rotation curve of $v_{\text {rot }} \sim 150 \mathrm{~km} \mathrm{~s}^{-1}$, which is consistent with the rotation curves derived by Guharthakurta et al. (1988) and Sperandio et al. (1995).

\subsection{The non-collisional component}

The non-collisional component consists of 49125 particles, which simulate the galactic halo, bulge, and disk. The characteristics of the different galactic components are shown in Table 2. The particle trajectories are integrated using an adaptive timestep for each particle. This method is described in Springel et al. (2001). The following criterion for an individual timestep is applied:

$\Delta t_{\mathrm{i}}=\frac{20 \mathrm{~km} \mathrm{~s}^{-1}}{a_{\mathrm{i}}}$,

where $a_{\mathrm{i}}$ is the acceleration of the particle "i". The minimum of all $t_{\mathrm{i}}$ defines the global timestep used for the Burlisch-Stoer integrator that integrates the collisional component.

The setup of the initial conditions was made by the program described in Hernquist (1993) with the following parameters:

- halo: mass $M=1.5 \times 10^{11} M_{\odot}$, core radius $r_{\mathrm{c}}=3 \mathrm{kpc}$, tidal radius $r_{\mathrm{t}}=30 \mathrm{kpc}$, cutoff radius $R=90 \mathrm{kpc}$,

- bulge: mass $M=5.2 \times 10^{9} M_{\odot}$, scale length $l=0.3 \mathrm{kpc}$, cutoff radius $R=30 \mathrm{kpc}$,

- disk: mass $M=2.6 \times 10^{10} M_{\odot}$, scale length $l=3 \mathrm{kpc}$, cutoff radius $R=45 \mathrm{kpc}$, disk thickness $z_{0}=600 \mathrm{pc}$.

10000 collisional particles with a $1 / R$ column density profile were added and the system was evolved during 2 Gyr in order to obtain a relaxed system. At the end of the simulation the difference between the total energy and the total angular momentum of the system was smaller than $0.5 \%$ of their initial value. During the last Gyr of this simulation the disk properties (disk height, surface density profile, density profile) did not change within $5 \%$. The final state of this simulation was used as the initial state for the here presented simulations.

The companion galaxy with a mass of $10^{11} M_{\odot}$ is simulated by 11000 particles forming a Plummer sphere with with a core radius of $2 \mathrm{kpc}$. 
Table 3. Model parameters for time dependent ram pressure stripping.

\begin{tabular}{lr}
\hline \hline maximum rampressure $\left(\mathrm{cm}^{-3}\left(\mathrm{~km} \mathrm{~s}^{-1}\right)^{2}\right)$ & 5000 \\
inclination angle between orbital and disk plane & $0^{\circ}$ \\
final HI deficiency & 0.1 \\
\hline
\end{tabular}

\section{The simulations}

I have made four different simulations using different interactions and different methods to model the galactic components (dark halo, stars, and gas).

1. Time dependent ram pressure stripping, no gravitational interaction. The gravitational potential of the galaxy is fixed and analytically given.

2. Time dependent ram pressure stripping, no gravitational interaction. The gravitational potential of the galaxy is simulated by non-collisional particles.

3. Gravitational interaction, no ram pressure stripping. The gravitational potential of the galaxy is simulated by noncollisional particles.

4. Gravitational interaction and constant ram pressure. The gravitational potential of the galaxy is simulated by noncollisional particles.

\section{Time dependent ram pressure stripping}

In Vollmer et al. (2001) we simulated different galaxy orbits with different inclination angles $i$ between the orbital and the disk plane. Phookun \& Mundy (1995) suggested that NGC 4654 is moving edge-on through the ICM. Indeed, only simulations with $i<20^{\circ}$ show an extended tail several $10^{8} \mathrm{yr}$ after the galaxy's closest passage to the cluster center. Furthermore, the simulation has to reproduce the observed $\mathrm{HI}$ deficiency of NGC 4654. With $i=0^{\circ}$ (edge-on stripping), a maximum ram pressure $p_{\text {ram }}^{\max }>5000 \mathrm{~cm}^{-3}\left(\mathrm{~km} \mathrm{~s}^{-1}\right)^{2}$ leads to a final Hi deficiency $D E F \geq 0.05$. It turned out that the best correspondence between the observed and the model Hi surface density exists for $p_{\text {ram }}=5000 \mathrm{~cm}^{-3}\left(\mathrm{~km} \mathrm{~s}^{-1}\right)^{2}$. In Vollmer et al. (2001) we have shown that the highest realistic maximum ram pressure is $p_{\text {ram }}^{\max } \sim 10000 \mathrm{~cm}^{-3}\left(\mathrm{~km} \mathrm{~s}^{-1}\right)^{2}$. In order to obtain higher values the galaxy would have to approach M87 to distances where a damaging gravitational interaction is ineluctable. Thus, values of the maximum ram pressure in the range $5000 \mathrm{~cm}^{-3}\left(\mathrm{~km} \mathrm{~s}^{-1}\right)^{2}<p_{\text {ram }}<10000 \mathrm{~cm}^{-3}\left(\mathrm{~km} \mathrm{~s}^{-1}\right)^{2}$ and the inclination angles in the range of $0^{\circ} \leq i \leq 10^{\circ}$ can reproduce the Hi observations. Since the observed Hi deficiency has an error of \pm 0.2 , the final model deficiency of $D E F=0.05$ is consistent with the observed value $(D E F=0.17)$, i.e. no $\mathrm{HI}_{\mathrm{I}}$ deficiency. The best fit model parameters are given in Table 3.

\subsection{The projection on the sky}

In order to project the model gas distribution on the sky, three angles are needed: the position angle, the inclination angle, and the azimuthal angle within the plane of the galactic disk. The position angle can be determined observationally. If one

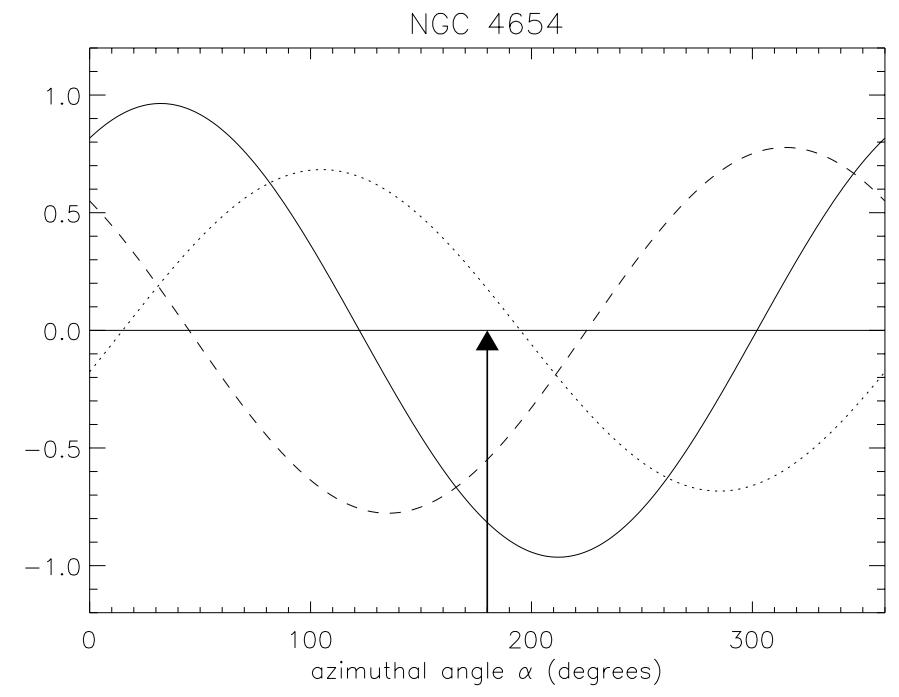

Fig. 1. The three components of the galaxy's velocity vector $v^{\text {gal }}$ as a function of the azimuthal angle $\alpha$. Solid: $v_{1}^{\text {gal }}$, dotted: $v_{2}^{\text {gal }}$, dashed: $v_{3}^{\text {gal }}$. The chosen angle is marked by an arrow. $v_{1}^{\text {gal }}$ is the component parallel to the axis of right ascension (positive is to negative right ascensions), $v_{2}^{\text {gal }}$ is that parallel to the axis of declination, and $v_{3}^{\text {gal }}$ is that parallel to the line of sight.

assumes that the spiral arms of NGC 4654 are trailing, its velocity field (Phookun \& Mundy 1995) places its northern edge in front of the galaxy center, i.e. the sign of the inclination angle $i$ is known.

In general, galaxies have trailing spiral arms. Galaxies with leading spiral arms are assumed to have undergone a plunging retrograde encounter with another galaxy (see, e.g., Thomasson et al. 1989 or Byrd et al. 1993), which should not be the case for NGC 4654. Since NGC 4654 harbors a bar and two normal spiral arms, there is no apparent reason why its spiral arms should be leading. It will be shown in Sect. 5 that the assumption of trailing spirals is important for the model.

With a given position angle (PA) and a given inclination angle $i$, only the azimuthal $(\alpha)$ in the galactic plane can be varied. I assume that the galaxy orbit within the cluster is approximately linear in space (see Vollmer et al. 2001). If NGC 4654 is emerging from the cluster core, it must be located in front of M87, because its radial velocity with respect to the cluster mean is negative $\left(\Delta v \sim-100 \mathrm{~km} \mathrm{~s}^{-1}\right)$. Furthermore, the component of its three-dimensional velocity vector parallel to the right ascension axis must be negative (the galaxy is moving to the east).

In Fig. 1 the three components of the galaxy's velocity vector $\boldsymbol{v}^{\text {gal }}$ are shown as a function of $\alpha$. $v_{1}^{\text {gal }}$ is the component parallel to the axis of right ascension (positive is to negative right ascensions), $v_{2}^{\mathrm{gal}}$ is that parallel to the axis of declination, and $v_{3}^{\text {gal }}$ is that parallel to the line of sight. The constraints $v_{3}^{\text {gal }}<0 \mathrm{~km} \mathrm{~s}^{-1}$ and $v_{1}^{\text {gal }}<0 \mathrm{~km} \mathrm{~s}^{-1}$ imply $130^{\circ} \leq \alpha \leq 220^{\circ}$. It turned out that $\alpha=180^{\circ}$ leads to the best fit to observations. This angle is marked by an arrow in Fig. 1. 

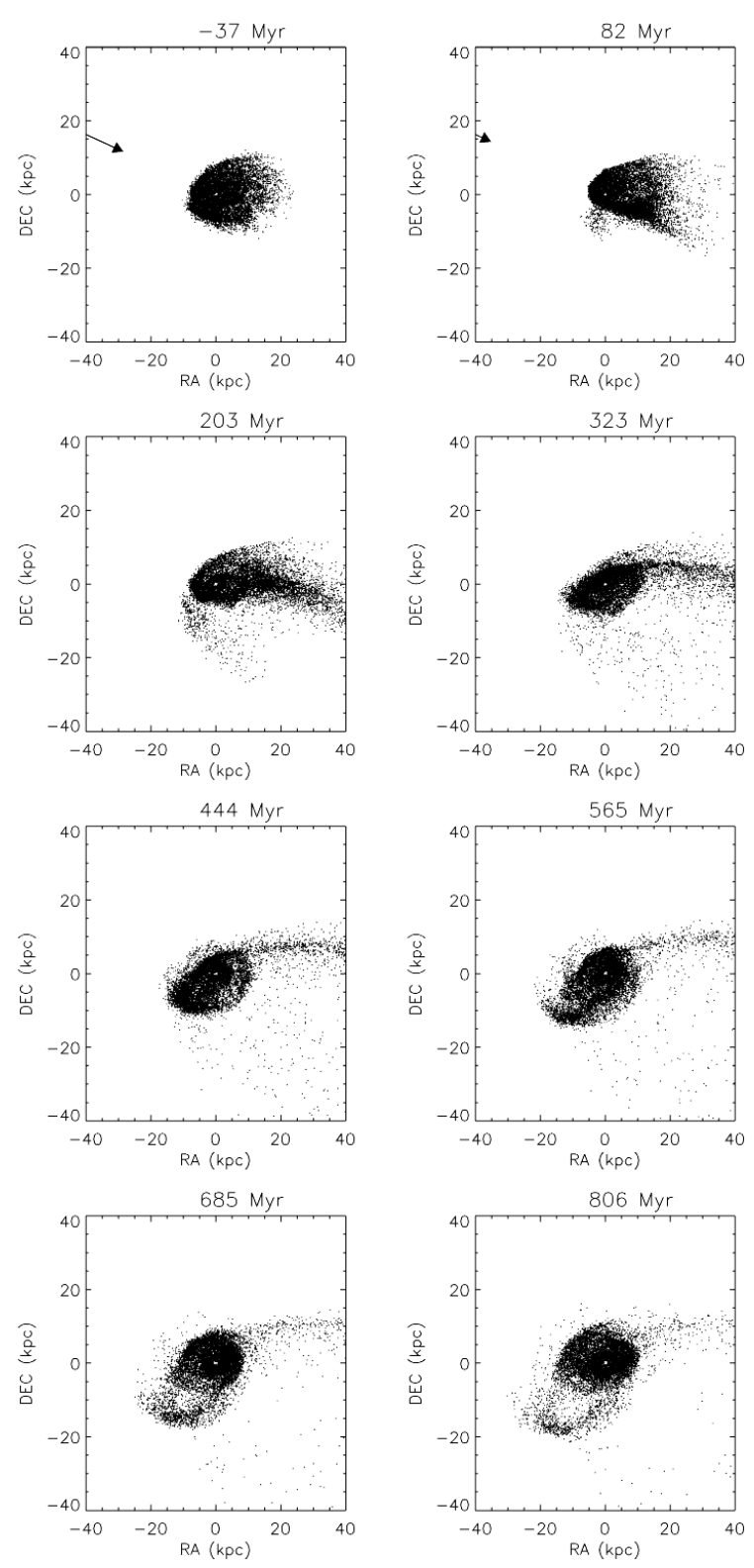

Fig. 2. Snapshots of the evolution of the galaxy's ISM with a fixed gravitational potential. The $x$-axis corresponds to the right ascension, The $y$-axis to declination. The elapsed time is indicated at the top of each panel. The position and inclination angle of NGC 4654 are used. The galaxy rotates counter-clockwise. It is moving to the north-east, i.e. the wind is coming from the north-east (indicated by the arrows). The length of the arrow is proportional to the ram pressure $\left(\rho_{\mathrm{ICM}} v_{\mathrm{gal}}^{2}\right)$.

\subsection{The simulations}

Figures 2 and 3 show the evolution of the galaxy's ISM after its closest passage to the cluster center for the model with a fixed gravitational potential and a galaxy model including a non-collisional component respectively. The position and inclination angle of NGC 4654 are used. The time $t$ can be found above each frame. The closest passage to the cluster center corresponds to $t=0 \mathrm{yr}$. The arrow indicates the direction of the wind, i.e. it is opposite to the galaxy's motion within the cluster. The length of the arrows is proportional to the ram pressure $p_{\text {ram }}=\rho_{\mathrm{ICM}} v_{\text {gal }}^{2}$, where $\rho_{\mathrm{ICM}}$ is the ICM density and $v_{\text {gal }}$
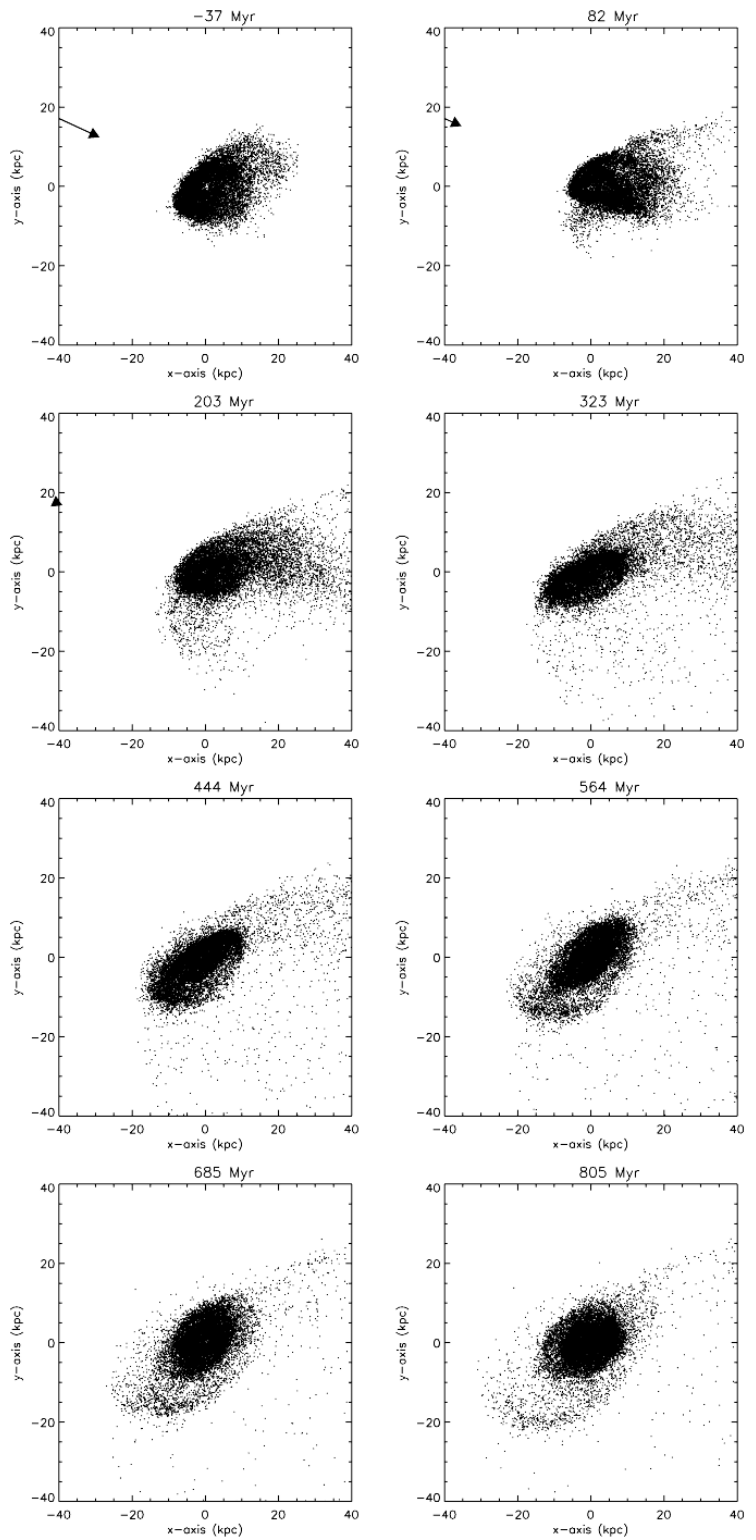

Fig. 3. Snapshots of the evolution of the galaxy's ISM for a galaxy model including a non-collisional component. The $x$-axis corresponds to the right ascension, The $y$-axis to declination. The elapsed time is indicated at the top of each panel. The position and inclination angle of NGC 4654 are used. The galaxy rotates counter-clockwise. It is moving to the north-east, i.e. the wind is coming from the north-east (indicated by the arrows). The length of the arrow is proportional to the ram pressure $\left(\rho_{\mathrm{ICM}} v_{\mathrm{gal}}^{2}\right)$.

is the galaxy velocity with respect to the cluster mean. The northern edge of the galaxy is in front of the galaxy center. The galaxy is rotating counter-clockwise. Since both simulations are very similar, I will describe in the following the main features for both simulations: at approximately maximum ram pressure $(t=0)$ an overdensity builds up in the direction of the galaxy's motion, where the gas has been pushed to smaller radii. At $t \sim 100 \mathrm{Myr}$ the wind has driven out the gas to the north where the vector of the galactic rotation velocity is parallel to the wind direction. In Vollmer et al. (2001) this is called the accelerated arm. A weak decelerated arm can also be seen 
in the south. At $t \sim 200 \mathrm{Myr}$ ram pressure has already ceased completely and the evolution of the galaxy is entirely due to rotation and re-accretion of the gas, which has not been accelerated to the escape velocity $\left(v_{\text {esc }} \sim \sqrt{2} v_{\text {rot }}\right)$. Due to rotation, the accelerated arm moves to the north and a part of its material falls back onto the galaxy. At $t>350 \mathrm{Myr}$ an asymmetric shell structure is building up in the south, which is most prominent in the south-east. This south-eastern part expands and forms an extended tail at $t \sim 700 \mathrm{Myr}$ after the galaxy has passed the cluster center.

The main difference of the gas dynamics between the two models is that the gravitational potential of model galaxy including a non-collisional component can change with time. The ISM-ICM interaction pushes the gas to smaller galactic radii and heats it. This process also heats the stellar disk. At maximum ram pressure the surface density in the north-east increase by a factor $\sim 2$ and $\sim 2.5 \times 10^{9} M_{\odot}$ of gas, which represents $\sim 10 \%$ of the total enclosed mass within $5 \mathrm{kpc}$, are displaced to the south-west. In addition, this gravitational perturbation triggers the formation of an asymmetry of the stellar distribution, which can also heat the stellar disk. Thus, the length scale of its gravitational potential in $z$ direction increases, i.e. the motion of the gas is less two dimensional than in the case of the fixed gravitational potential. The result is that the asymmetries of the gas distribution are smoothed out in the case of the model including a non-collisional component. In a real galaxy the gas might cool through shocks during the interaction. Moreover, the disk heating through the the heated gas might be a numerical artifact due to the relatively small number of particles used in the simulation. It is not clear if the gravitational heating is sufficient to produce the large scale height observed in the model.

There is another big difference between the two models: the disk and bulge stars react to the change of the gravitational potential when the gas is pushed to small galactic radii. This produces a gravitational shock, i.e. the stellar surface density also shows an asymmetry with a delay of $\sim 400 \mathrm{Myr}$. This asymmetric distribution rotates slowly and smears out within a few rotation times. Figure 4 shows the distribution of disk stars seen face-on and projected on the sky with the parameters of NGC 4654 at $t=800 \mathrm{Myr}$, i.e. the snapshot that I want to compare with observations. The bottom panel of Fig. 4 shows the $K$ band image of Boselli et al. (1997). Seen face-on, the stellar surface density of the outer disk $(R>6 \mathrm{kpc})$ is higher in the north than in the south. The stellar density in the inner disk is high with a strong decline at $R \sim 6 \mathrm{kpc}$. This translates into an elliptical projected stellar distribution with the galaxy center in the south-eastern focal point. This resembles the overall observed distribution of the old stellar component in $K$ band (Fig. 4 bottom panel). Thus the observed overall asymmetry of the stellar distribution can be reproduced by a gravitational shock due to edge-on ram pressure stripping, but it is not possible to create a bar nor spiral arms in this way.

Figure 5 shows the stellar distribution and its velocity field at $t=800 \mathrm{Myr}$ projected on the sky with the parameters of NGC 4654. The velocity field shows clearly a steepening in the north-west, whereas the southern part of the galaxy has the velocity field of a rotation curve that flattens in the outer disk.
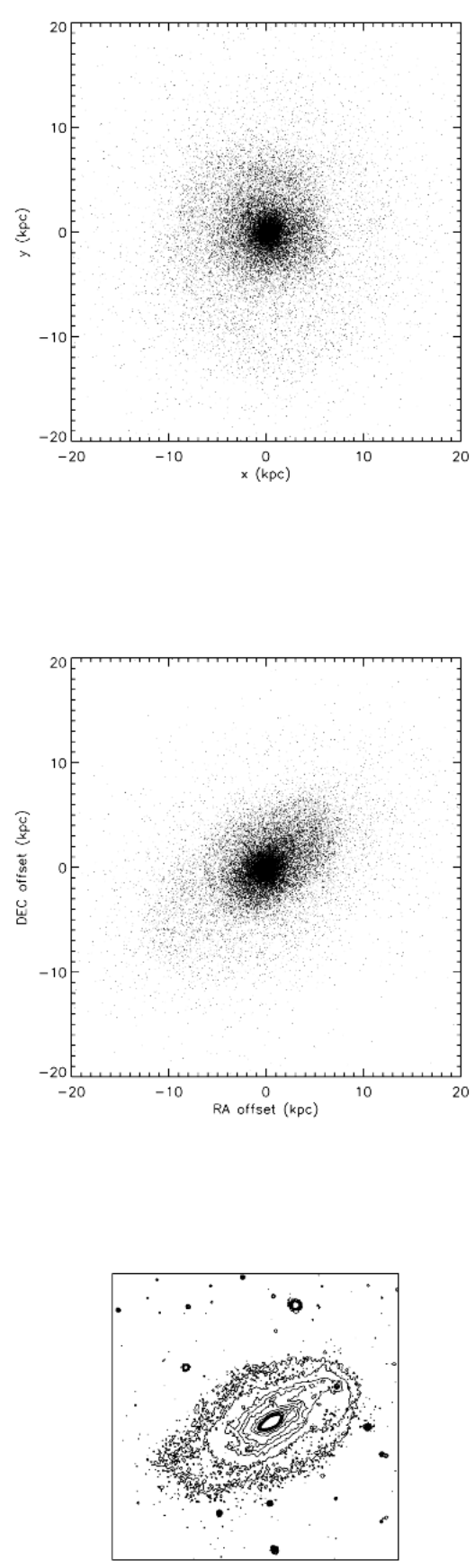

Fig. 4. Top panel: model distribution of the disk stars at $t=800 \mathrm{Myr}$ seen face-on. Middle panel: model distribution of the disk stars at $t=800 \mathrm{Myr}$ projected on the sky with the PA and $i$ of NGC 4654 . Bottom panel: $K$ band image of NGC 4654 (Boselli et al. 1997).

\subsection{Comparison between observations and simulations}

In this section the gas distribution and the velocity field of NGC 4654 are directly compared to the last model snapshots of Figs. 2 and 3, i.e. at $t=800 \mathrm{Myr}$.

Figure 6 shows the Hi distribution of NGC 4654 (bottom panel) together with the model gas distributions convolved to the beamsize of the $21-\mathrm{cm}-$ line observations $\left(25^{\prime \prime}\right)$. The top panel shows the gas distribution of the model with a fixed gravitational potential, the middle panel shows that of the model 

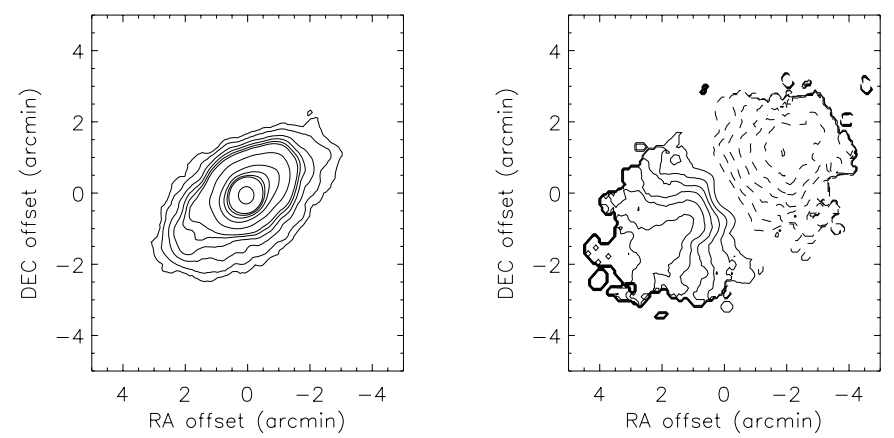

Fig. 5. Left panel: model stellar distribution at $t=800$ Myr. Right panel: model stellar velocity field. Both distributions are projected on the sky using PA and $i$ of NGC 4654 .

including a non-collisional component. The main characteristics of the observed gas distribution (Fig. 6 bottom panel) are (i) the prominent maximum at the north-western edge of the disk, (ii) the overall asymmetry within the disk with more gas in the south-east, (iii) the linear extended tail to the south-east. The model using a fixed gravitational potential (Fig. 6 top panel) can reproduce feature (i) and (ii). It also shows an extended tail to the south-east, but it has a higher column density, is curved, and is much less extended than the observed tail. The model including a non-collisional component (Fig. 6 middle panel) fails in reproducing feature (i) and (ii), but can reproduce feature (iii), i.e. the extended, low column density, south-eastern tail. However, the model tail is slightly curved. The low column density of this tail is due to the tidal heating of the disk during the ICM-ISM interaction (see Sect. 4.2).

The Hi velocity fields of the two models and the observed velocity field can be seen in Fig. 7. The main characteristics of the observed Hi velocity field (Fig. 7 bottom panel) are (i) the plateau of constant rotation velocity in the south-east, (ii) the steepening of the rotation curve in the north west, (iii) the structure of the velocity in the extended south-eastern tail. The model using a fixed gravitational potential (Fig. 7 top panel) shows the features (i) and (ii), but fails in reproducing feature (iii). The model including a non-collisional component (Fig. 7 middle panel) shows a less clear plateau, but feature (ii) is visible. It also fails in reproducing the observed velocity field in the extended south-eastern tail.

In order to compare the characteristic structure of the $\mathrm{HI}$ velocity fields, the position-velocity diagrams along (i) the major axis, (ii) the tail, and (iii) the minor axis are shown in Figs. 8, 9 , and 10 , respectively. The model cut for a fixed gravitational potential along the major axis (Fig. 8, top panel) nicely shows the observed plateau of constant rotation velocity. At the opposite side, the main component is spatially reduced with respect to the side with positive offsets and shows a constant rotation. Thus, this simulation is able to reproduce the south-eastern part of the position-velocity diagram, but not the north-western part.

The cut for the model including a non-collisional component (Fig. 8, middle panel) also shows a plateau at the southeastern side (negative offsets) that is spatially reduced having only one third of the extent of the observed plateau. The linear rise at the north-western side is reproduced. At the
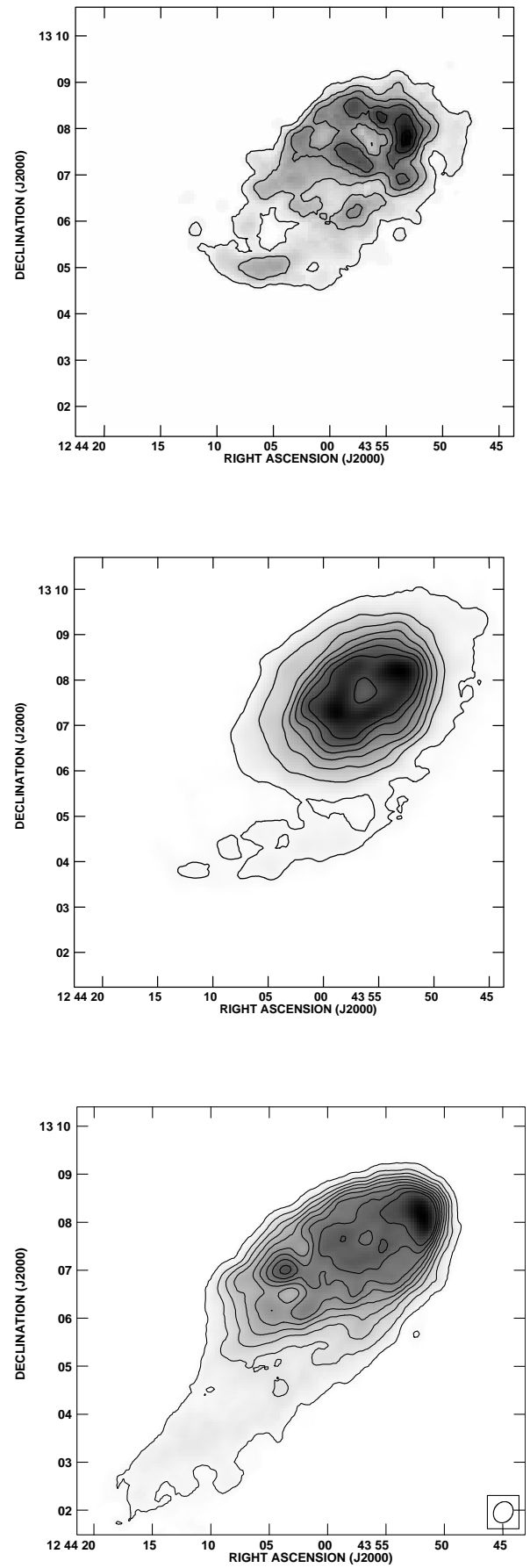

Fig. 6. Top panel: model distribution of the Hi gas using a fixed gravitational potential. The contours correspond to $(1,5,9,13,17,21$, $25) \times 10^{19} \mathrm{~cm}^{-2}$. Middle panel: distribution of the $\mathrm{HI}$ gas using the model including a non-collisional component with the same contour levels. Bottom panel: Hi distribution of NGC 4654 (Phookun \& Mundy 1995) with the same contour levels.

north-western edge the rotation curve is falling again producing closed contours in the velocity field. This is due to the velocity dispersion of the gas within the disk, which is higher than that of the observed galaxy, because there is no explicit cooling mechanism included in the model.

The model cuts through the tail (Fig. 9, top and middle panel) are very similar for both models. As already seen above, 

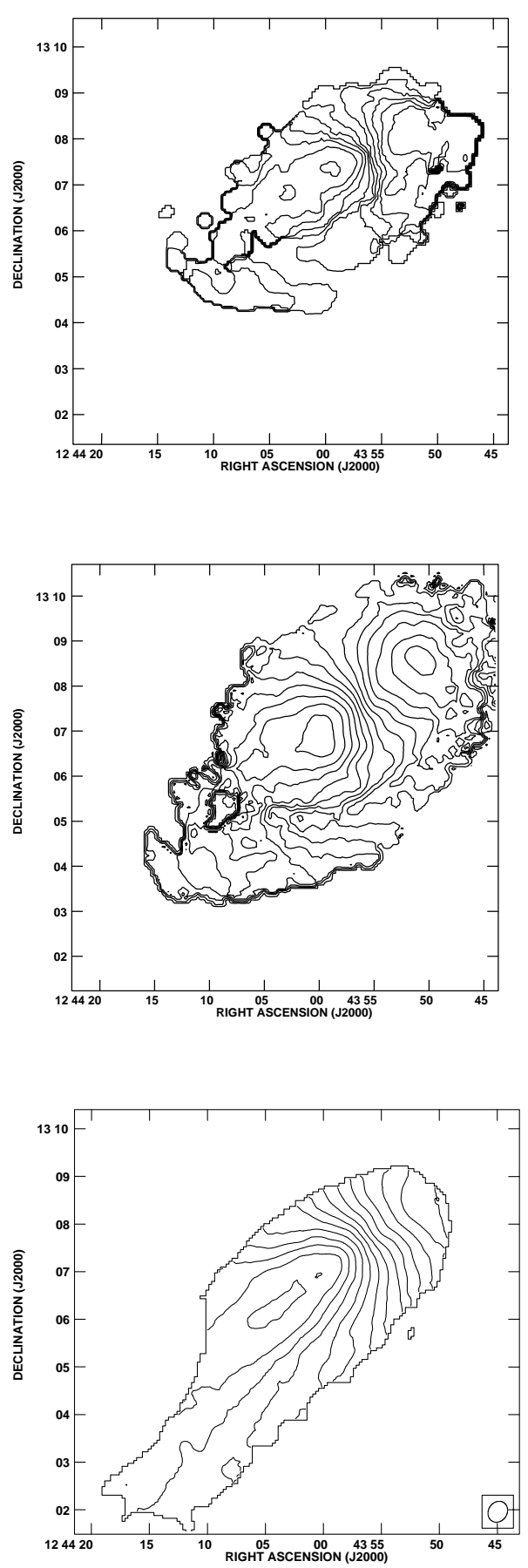

Fig. 7. Top panel: model Hi velocity field using a fixed gravitational potential. The contours, in $\mathrm{km} \mathrm{s}^{-1}$, are from 900 (north-west) to 1160 (south-east) in steps of 20 for both velocity fields. Middle panel: gas velocity field using the model including a non-collisional component with the same contour levels. Bottom panel: Hi velocity field of NGC 4654 (Phookun \& Mundy 1995) with the same contour levels.

these model cuts differ from the observed one in the sense that the velocity structure of the model tail is almost linear, whereas that of the observed tail is significantly curved.

The model cuts along the minor axis (Fig. 10) shows qualitatively the same deviations from circular rotation as the cut through the observed data cube.
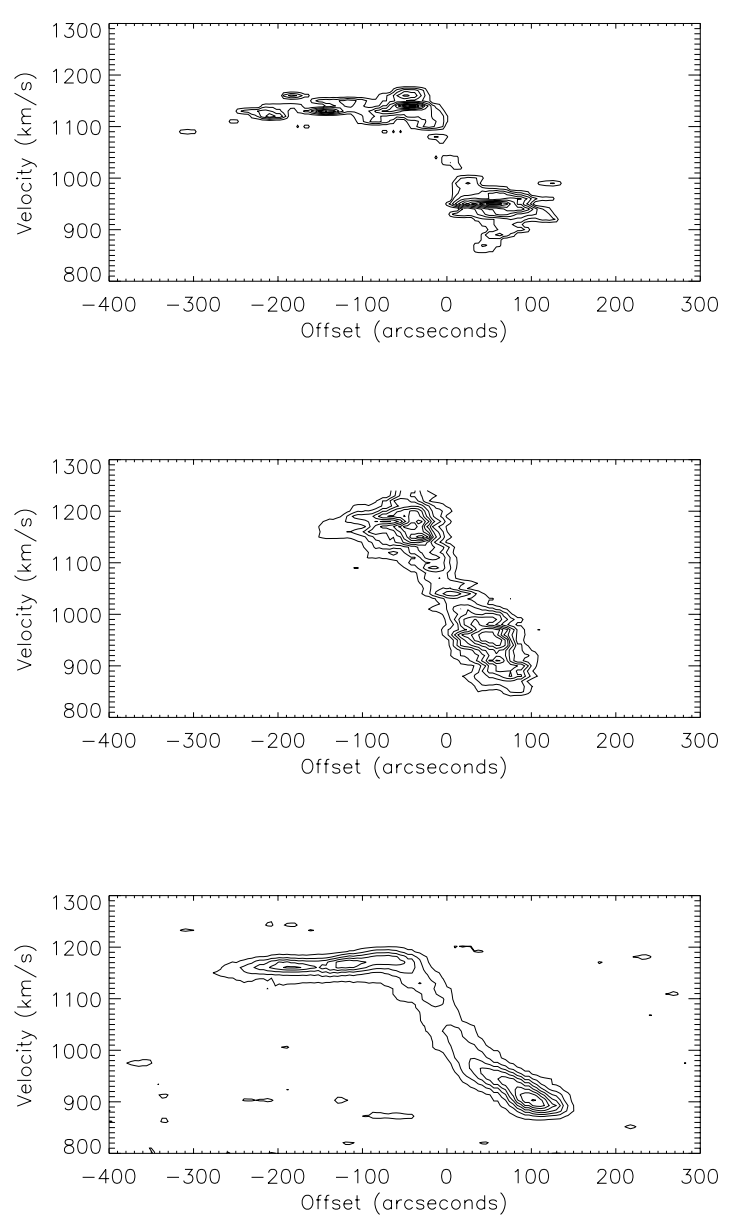

Fig. 8. Position-velocity cut through the Hi cube along the major axis. Top panel: model using a fixed gravitational potential. Middle panel: model including a non-collisional component. Bottom: Hi (Phookun \& Mundy 1995).

\section{The active stripping scenario}

The simulations of Sect. 4.2 show that ram pressure pushes gas beyond the optical radius of the galaxy at $t>0 \mathrm{Myr}$. A taillike structure is built up at even later times, clearly after the galaxy's closest passage to the cluster center. This result rules out the scenario of active ram pressure stripping.

Nevertheless, if one assumes that the efficiency of ram pressure to push the atomic gas has been greatly underestimated and that the galaxy is moving through a region of enhanced ICM density, it is possible to compare another simulation snapshot with the observations, where ram pressure stripping is still active. I have chosen the following simulation parameters to best reproduce the observed $\mathrm{HI}$ distribution with the extended tail: maximum ram pressure $p_{\text {ram }}=2000 \mathrm{~cm}^{-3}\left(\mathrm{~km} \mathrm{~s}^{-1}\right)^{2}$, and $i=0^{\circ}$ (edge-on stripping). The timestep of the snapshot is $t \sim 100 \mathrm{Myr}$, i.e. ram pressure is still active. The gas distribution and velocity field of this snapshot can be seen in Fig. 11. The tail represents the accelerated arm (cf. Fig. 2). The assumption that the spiral arms are trailing places it imperatively in the north (the galaxy rotates counter-clockwise). The column density is uniform over the whole length of the tail, which is not observed. Concerning the velocity field, the southern plateau 

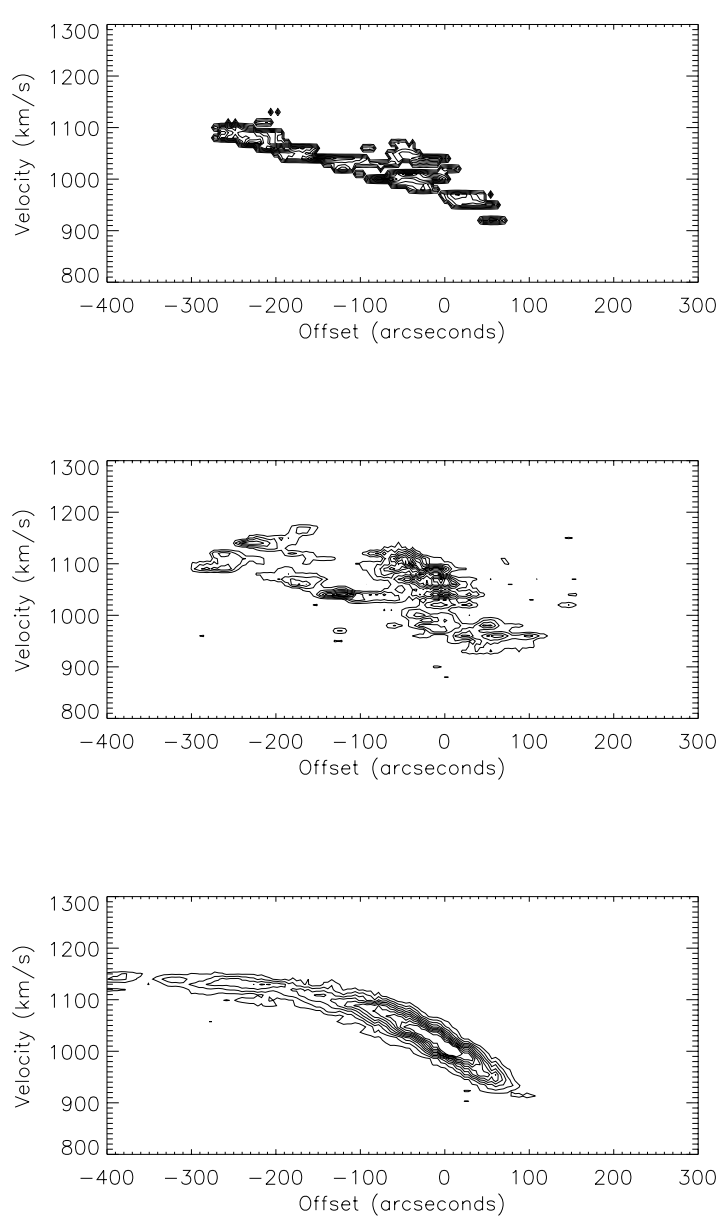

Fig. 9. Position-velocity cut through the Hi cube along the tail. Top panel: model using a fixed gravitational potential. Middle panel: model including a non-collisional component. Bottom: Hi (Phookun \& Mundy 1995).

of constant velocity is inclined with respect to the optical major axis. Thus, this snapshot clearly does not fit the Hi observations appropriately.

If one goes still further and drops the assumption that the spiral arms of NGC 4654 are trailing (Sect. 4.1), the accelerated arm in Fig. 11 would be located in the south as observed. In this case, two discrepancies between the model and the observations persist: (i) the uniform column density of the model tail and (ii) the inclination of the southern plateau of constant rotation velocity of the model.

\section{Gravitational interaction}

\subsection{Gravitational interaction alone}

In this simulation we assume that NGC 4654 has been gravitationally perturbed by its companion NGC 4639. Since we need a close encounter in order to generate the observed asymmetry of the stellar disk of NGC 4654 without disrupting it, I choose a retrograde encounter. The perturbing galaxy is modeled as a plummer sphere with a mass of $10^{11} M_{\odot}$ and a length scale of $2 \mathrm{kpc}$. The impact parameter is $21 \mathrm{kpc}$ and the maximum relative velocity is $350 \mathrm{~km} \mathrm{~s}^{-1}$. The minimum distance in the plane of the disk of NGC 4654 is $18 \mathrm{kpc}$. I assume here that the
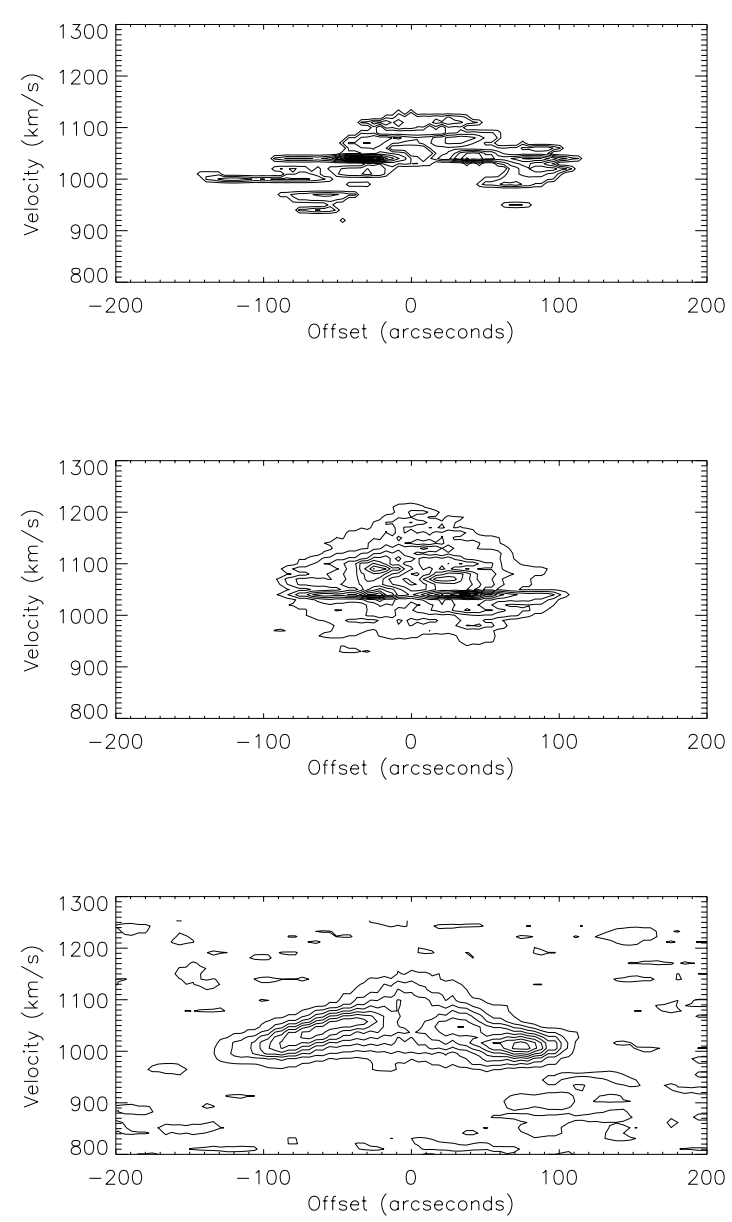

Fig. 10. Position-velocity cut through the Hi cube along the minor axis. Top panel: model using a fixed gravitational potential. Middle panel: model including a non-collisional component. Bottom: $\mathrm{H}_{\mathrm{I}}$ (Phookun \& Mundy 1995).

inclination angle of the disk of NGC 4639 is such that there is no ISM-ISM interaction.

I define the following coordinate system: $x-y$ plane: disk plane, $z$ axis: perpendicular to the disk plane. The origin is the center of NGC 4654. In this system the initial conditions for NGC 4639 are: $x=-40 \mathrm{kpc}, y=-70 \mathrm{kpc}, z=70 \mathrm{kpc}, v_{x}=$ $0 \mathrm{~km} \mathrm{~s}^{-1}, v_{y}=130 \mathrm{~km} \mathrm{~s}^{-1}, v_{z}=-130 \mathrm{~km} \mathrm{~s}^{-1}$. It is marked as a cross on the snapshots.

At $t=1 \mathrm{Gyr}$, the projected distance and radial velocity difference with respect to NGC 4654 using the PA and $i$ of NGC 4654 are: $\tilde{x}=60 \mathrm{kpc}, \tilde{y}=55 \mathrm{kpc}, v_{\mathrm{r}}=70 \mathrm{~km} \mathrm{~s}^{-1}$. This compares to the observed projected position and radial velocity with respect to NGC 4654 of $\tilde{x}_{\mathrm{obs}}=80 \mathrm{kpc}, \tilde{y}_{\mathrm{obs}}=39 \mathrm{kpc}$, $v_{\mathrm{r}}^{\mathrm{obs}}=-35 \mathrm{~km} \mathrm{~s}^{-1}$. Both, positions and radial velocity, are close enough to suppose that the simulation should show the main characteristics of a possible gravitational interaction between NGC 4654 and NGC 4639.

Figure 12 shows eight timesteps of this simulation. The timestep of closest encounter is $t=460 \mathrm{Myr}$. After the passage of NGC 4639, NGC 4654 begins to form a large bar with two asymmetric spiral arm, the south-eastern being more extended than the north-western arm. After $\sim 350 \mathrm{Myr}$ these large scale spirals begin to wind up. A small, negligible number of stellar 

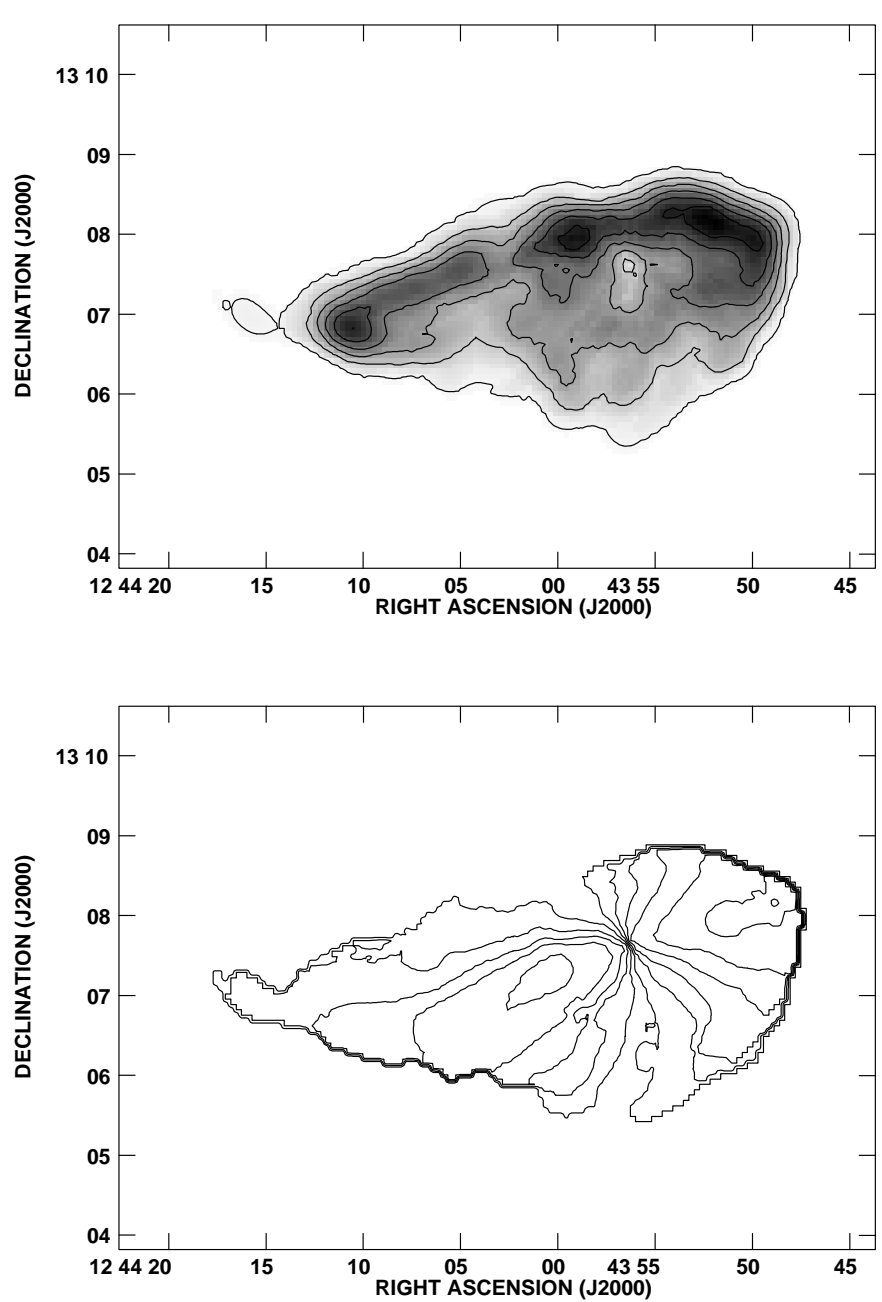

Fig. 11. Top: gas distribution of a simulation snapshot at $t \sim 100 \mathrm{Myr}$ with a maximum ram pressure of $2000 \mathrm{~cm}^{-3}\left(\mathrm{~km} \mathrm{~s}^{-1}\right)^{2}$ and $i=0^{\circ}$ (edge-on stripping). The contours correspond to $(1,5,9,13,17,21$, $25) \times 10^{19} \mathrm{~cm}^{-2}$. Bottom: corresponding velocity field. The contours, in $\mathrm{km} \mathrm{s}^{-1}$, are from 940 (north-west) to 1140 (south-east) in steps of 20.

particles is initially located outside the gas disk. They are responsible for the low density outer spiral arms for $t>700 \mathrm{Myr}$ that are not present in the gas distribution. On the other hand, the gas distribution has a $1 / R$ profile, whereas the stellar distribution has an exponential profile. This causes the difference between the high column density structure of the gaseous and stellar distribution. In the inner part of the galaxy, i.e. within its optical radius, a bar with two asymmetric spiral arms survive until $t=1 \mathrm{Gyr}$, i.e. $\sim 550 \mathrm{Myr}$ after the closest encounter. The north-western spiral arm is much tighter than the southwestern one as observed for NGC 4654 in the optical. Figure 13 shows a closer view of the stellar disk at $t=1 \mathrm{Gyr}$. The bar and the asymmetric spiral arms can be recognized. This morphology resembles closely that observed in the $K$ band (Fig. 4, bottom panel).

\subsection{Gravitational interaction and ram pressure}

As a next step, ram pressure is included in the model: $p_{\text {ram }}=$ $m_{\mathrm{p}} n_{\mathrm{ICM}} v_{\text {gal }}^{2}$, where $m_{\mathrm{p}}$ is the proton mass, $n_{\mathrm{ICM}}$ the density of the intracluster medium, and $v_{\text {gal }}$ the velocity of the galaxy within the cluster. I have chosen it to be constant in order to keep the model as simple as possible. The values for the density of the intracluster medium and the galaxy's velocity are typical for a galaxy located at the projected distance of NGC 4654, i.e. $n_{\mathrm{ICM}}=2 \times 10^{-4} \mathrm{~cm}^{-2}$ and $v_{\mathrm{gal}}=1000 \mathrm{~km} \mathrm{~s}^{-1}$. The direction of the galaxy's motion is parallel to its major axis and is pointing to the north-west. The perturbing mass simulating NGC 4639 has the same orbits as described in Sect. 6.

Figure 14 shows eight timesteps of this simulation.

Already at $t=300 \mathrm{Myr}$ ram pressure pushes the gas to the south-east of the galaxy that has been forced to larger galactic radii due to the tidal interaction with the perturbing mass. It forms a gaseous spiral arm at the western side of the galaxy at $t=400 \mathrm{Myr}$ which then moves to the north. It arrives at the northern edge of the galaxy at $t=700 \mathrm{Myr}$ and proceeds further to the south-east, where it forms an extended tail structure at $t=1 \mathrm{Gyr}$, which is corresponds to the observed tail of NGC 4654. As expected, the stellar distributions at this final timestep for the simulation with and without ram pressure (Figs. 14 and 12) are the same since the gas displacement is not important enough to trigger a strong stellar asymmetry as observed in Sect. 4.3.

\subsection{Comparison between observations and simulations}

In this section the gas distribution and the velocity field of NGC 4654 are directly compared to the last model snapshots of Figs. 12 and 14, i.e. at $t=1 \mathrm{Gyr}$.

Figure 15 shows the Hi distribution of NGC 4654 (bottom panel) together with the model gas distributions convolved to the beamsize of the $21-\mathrm{cm}-$ line observations $\left(25^{\prime \prime}\right)$. The top panel shows the gas distribution of the model of a gravitational interaction alone, the middle panel shows that a gravitational interaction together with constant ram pressure. For both models the asymmetry of the gas distribution within the optical radius along the major axis can be reproduced. The observed local maximum in the north-west as well as the extended region in the south-east are clearly visible in both model snapshots. The simulations using only ram pressure as perturbation could not reproduce these features (see Fig. 6).

The very extended south-eastern tail can only be reproduced by the model including ram pressure.

The velocity fields of the two models and the observed velocity field can be seen in Fig. 16.

In order to compare the characteristic structure of the velocity fields, the position-velocity diagrams along (i) the major axis, (ii) the tail, and (iii) the minor axis are shown in Figs. 17, 18 , and 19, respectively. The south-eastern side (negative offsets) of the model cuts along the major axis are very similar for both models, i.e. the dynamics of this part of the disk are not affected by ram pressure. The model cuts show a maximum at an offset of $\sim-50^{\prime \prime}$. The rotation curve then decreases slightly. The observed rotation curve does not show a maximum. It reaches the plateau of constant rotation velocity at an offset of $-40^{\prime \prime}$. The north-western side (positive offsets) of the 

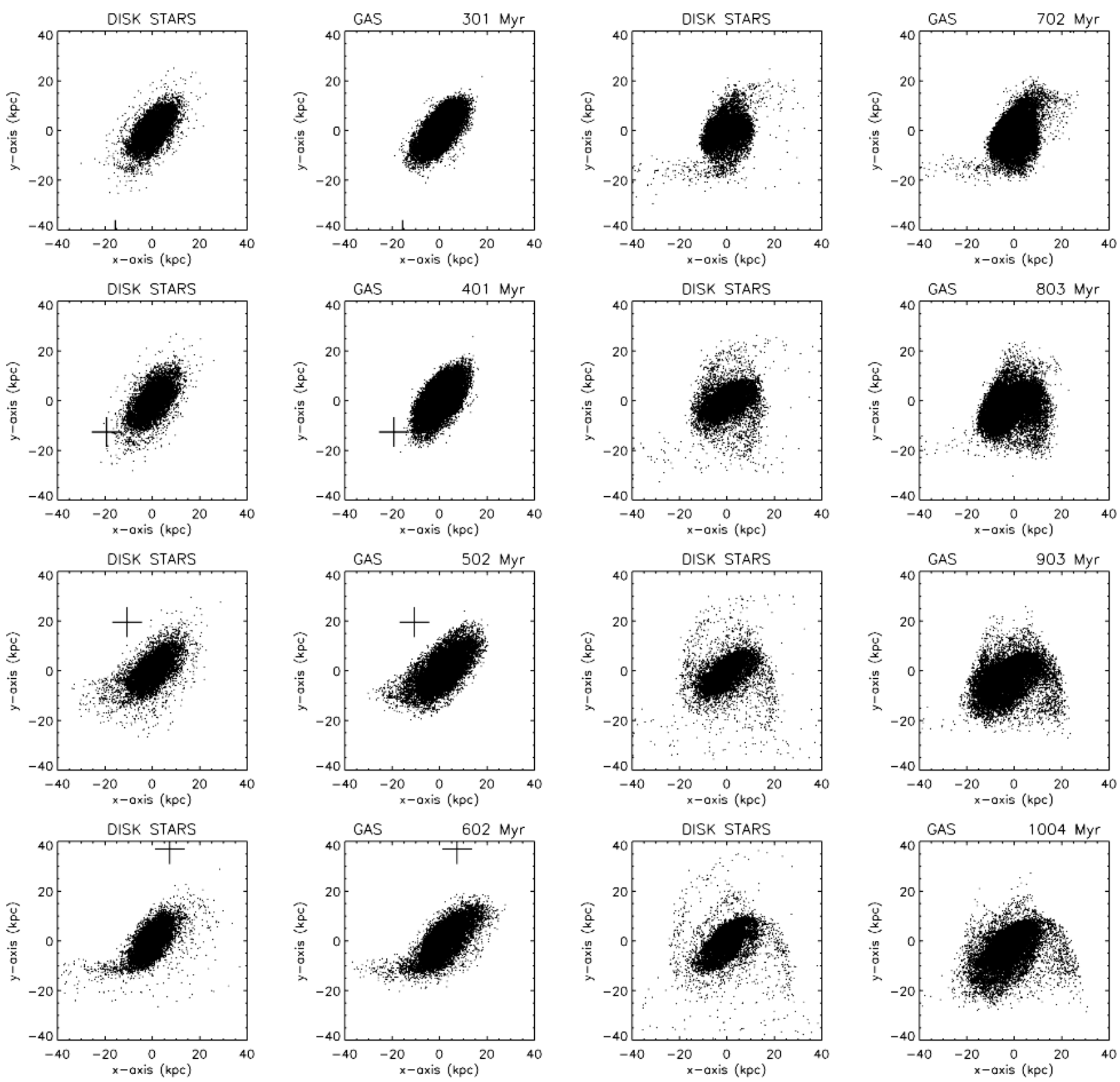

Fig. 12. Snapshots of the tidal interaction between NGC 4654 and NGC 4639. The left panels show the stellar disk, the right panels show the gas. The timesteps are marked on the upper right of each panel showing the gas. NGC 4639 is marked as a cross.

galaxy shows a slightly rising rotation curve for the model including only a gravitational interaction. In contrast, the model of the mixed interaction (gravitational and ram pressure) leads to a linear rising rotation curve in the north-west. Again, at the north-western edge the rotation curve is falling due to the velocity dispersion of the gas within the disk, which is higher than that of the observed galaxy, because there is no explicit cooling mechanism included in the model (cf. Sect. 4.3).

Since the model of a mixed interaction and observations show a constant rotation curve at one side and a rising rotation curve at the other side, I conclude that the main characteristics of the observed rotation curve are reproduced by this model.

The $\mathrm{p}-\mathrm{V}$ cuts along the tail reflect the structure of the gas distributions (Fig. 15). The gas distribution of the model without ram pressure extends further to the north-west (positive offsets) than the one including ram pressure. At the opposite side the situation is reversed. The model including ram pressure reproduces nicely the observed $\mathrm{p}-\mathrm{V}$ cut along the tail. The $\mathrm{p}-\mathrm{V}$ cuts of both models along the minor axis are very similar. They show a smaller but visible degree of asymmetry compared to the observed asymmetry.

\section{Discussion}

The main differences between the two models using ram pressure as the only perturbation are that (i) the disk is heated during the ICM-ISM interaction and (ii) the stellar distribution reacts to the heavily perturbed gas distribution. Whereas (ii) is 


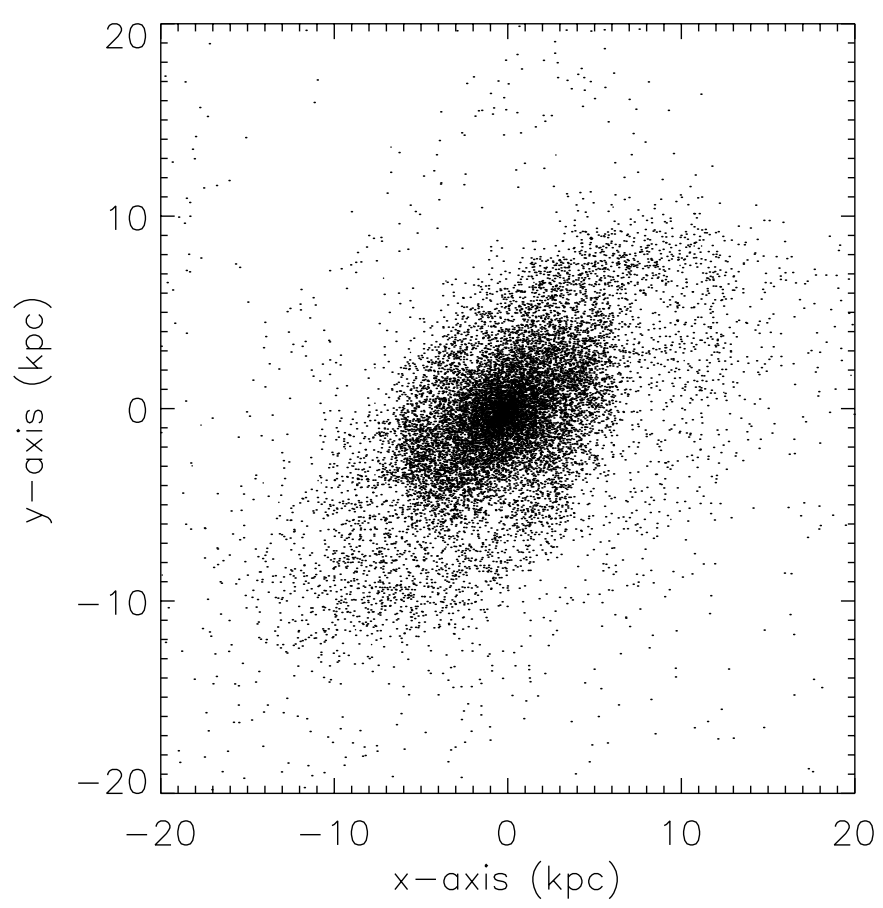

Fig. 13. Close-up of the stellar disk of the final snapshot after $1 \mathrm{Gyr}$ (see Fig. 12).

a robust result, the disk heating might be artificial, because of the discreteness of the model and because there is no gas cooling included in the model. Thus, the decrease of the gas column density in the extended tail has to be interpreted with caution. If one wants to compare this kind of models to observations of other galaxies, both models have to be taken into account. The situation is still more complicated by the fact that at the outer regions of the gas distribution evaporation effects might play an important rôle that can in principle lower the gas column density.

The comparison between the models and observations shows important differences. Whereas the model using ram pressure as the only perturbation can explain the observed asymmetric rotation curve and the extended, low surface density tail of NGC 4654, it cannot reproduce the observed velocity structure of the extended, south-eastern tail. Nevertheless, it is worth noting that a past, strong ram pressure event can produce a tail-like structure even $800 \mathrm{Myr}$ after the galaxy's closest passage to the cluster center. Since we observe a reaction of the stellar disk to the strongly perturbed gas distribution, the perturbation might be longer-lived than expected if one takes only the timescale of gas diffusion into account. Moreover, it is not possible to form a bar and asymmetric spirals as observed with ram pressure as the only perturbation.

The model using a gravitational interaction is able to reproduce the observed stellar distribution of NGC 4654. The chosen orbit of the perturbing galaxy, NGC 4639, is not unique. It has been chosen such that (i) the position of the perturber and its velocity are close to those observed for NGC 4639 and (ii) to reproduce the asymmetry of the stellar distribution. The aim of this work is not to explain all details of the observations, but to reproduce the main characteristics of the observations. It becomes clear that only the combination of a gravitational interaction and ram pressure can account for all characteristics. I want to stress here that it is important to compare the gas distribution and the gas kinematics. In the case of NGC 4645 it is the gas kinematics that discriminate between the models. Since the tidal interaction pulls gas to larger galactic radii, only a small amount of ram pressure is needed to produce the very extended gas tail.

Despite the fact that only the mixed perturbation (gravitational and ram pressure) can explain all characteristics of the observations, there are two potential problems with this model:

- Is an impact parameter between the two galaxies of $\sim 20 \mathrm{kpc}$ realistic? In this case the disk of NGC 4639 has to be almost parallel to that of NGC 4654 during the encounter to avoid an ISM-ISM interaction, which would complicate the scenario. This can be verified by making a model using two galaxy models that include a collisional and a noncollisional component. This introduces a further open parameter, i.e. the inclination angle of disk of NGC 4639 with respect to the disk of NGC 4654.

- In the restframe of NGC 4654, NGC 4639 has a velocity in the plane of the sky of $v_{\mathrm{N} 4639} \sim 145 \mathrm{~km} \mathrm{~s}^{-1}$ towards the north-west. With respect to the cluster both galaxies have a velocity of $\sim 1000 \mathrm{~km} \mathrm{~s}^{-1}$ mainly towards the cluster center (M87). Thus the atomic gas outside the optical radius of NGC 4639 should be affected by ram pressure in the same way as the gas of NGC 4654, i.e. it should be mainly located to the south-east of the galaxy center. Warmels (1988) observed indeed extended gas at the east of NGC 4639. However, this is not observed by Cayatte et al. (1990). They found an external emission region in the south-west of the galaxy center. Moreover Phookun \& Mundy (1995) did not detect any emission in the east outside the optical radius of NGC 4639. Since NGC 4639 is located at the edge of the primary beam of Cayatte et al. (1990) and Phookun \& Mundy (1995) their results have to be confirmed by observations especially dedicated to NGC 4639.

\section{Summary and conclusions}

Numerical models including ram pressure and gravitational interaction are compared to high-sensitivity Hi observations of the spiral galaxy NGC 4654 in the Virgo cluster. This galaxy shows a very extended, low surface density tail that does not show clear signs of rotation.

Four different models are presented, in order to investigate the origin of this tail:

- fixed gravitational potential of the galaxy, ram pressure is included,

- stellar content and dark halo simulated by a non-collisional component, ram pressure is included,

- gravitational interaction,

- gravitational interaction, ram pressure is included. 

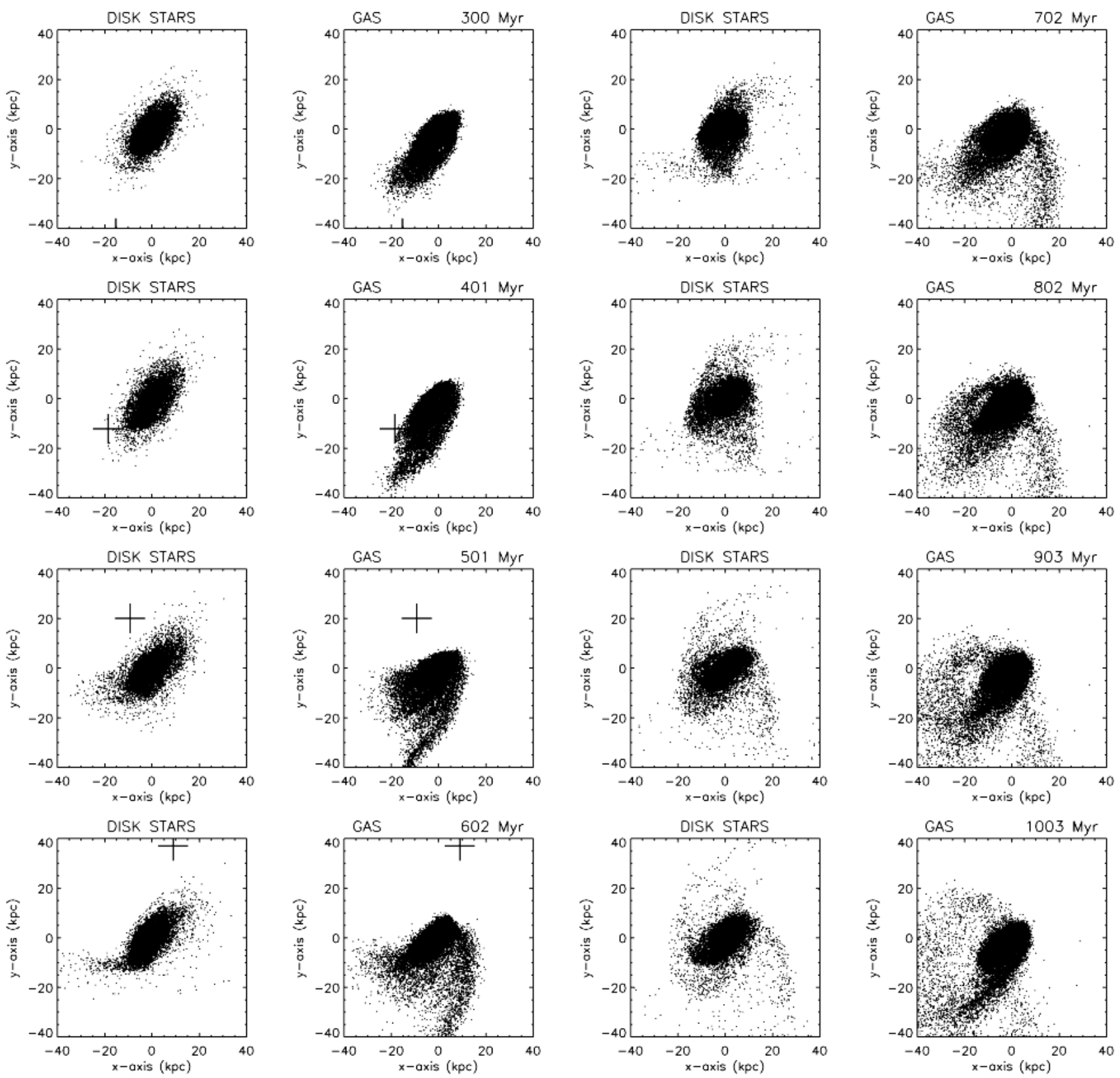

Fig. 14. Snapshots of the tidal interaction between NGC 4654 and NGC 4639 including a constant ram pressure. The left panels show the stellar disk, the right panels show the gas. The timesteps are marked on the upper right of each panel showing the gas. NGC 4639 is marked as a cross.

\subsection{Ram pressure as the only perturbation}

The temporal ram pressure profile corresponds to a realistic galaxy orbit within the cluster. Among the simulations of Vollmer et al. (2001) with different inclination angles $i$ between the disk and the orbital plane, one simulation was chosen, which reproduces the observed Hi deficiency and the Hi distribution. Limits for these parameters are: $5000 \mathrm{~cm}^{-3}\left(\mathrm{~km} \mathrm{~s}^{-1}\right)^{2}<$ $p_{\text {ram }}<10000 \mathrm{~cm}^{-3}\left(\mathrm{~km} \mathrm{~s}^{-1}\right)^{2}$ and $0^{\circ}<i<10^{\circ}$. The timestep of the snapshot compared to the observations is roughly given by the projected distance of NGC 4654 to the cluster center divided by $1700 \mathrm{~km} \mathrm{~s}^{-1}$, which represents a typical velocity (with respect to the cluster mean velocity) of a mildly stripped galaxy in the core of the Virgo cluster (Vollmer et al. 2001). With the given PA and inclination angle, the azimuthal angle has to be chosen such that the radial velocity of the model galaxy equals approximately that of NGC 4654. These are very severe constraints for the model, reducing the number of possible snapshots to a few.

\subsection{Gravitational interaction}

The parameters for the gravitational interaction were chosen such that (i) the observed position and radial velocity of NGC 4639 are reproduced and (ii) the observed asymmetry of the stellar content of NGC 4654 is reproduced. Asymmetric spiral arms without disrupting the galaxy can only be obtained with a very close, retrograde encounter. 

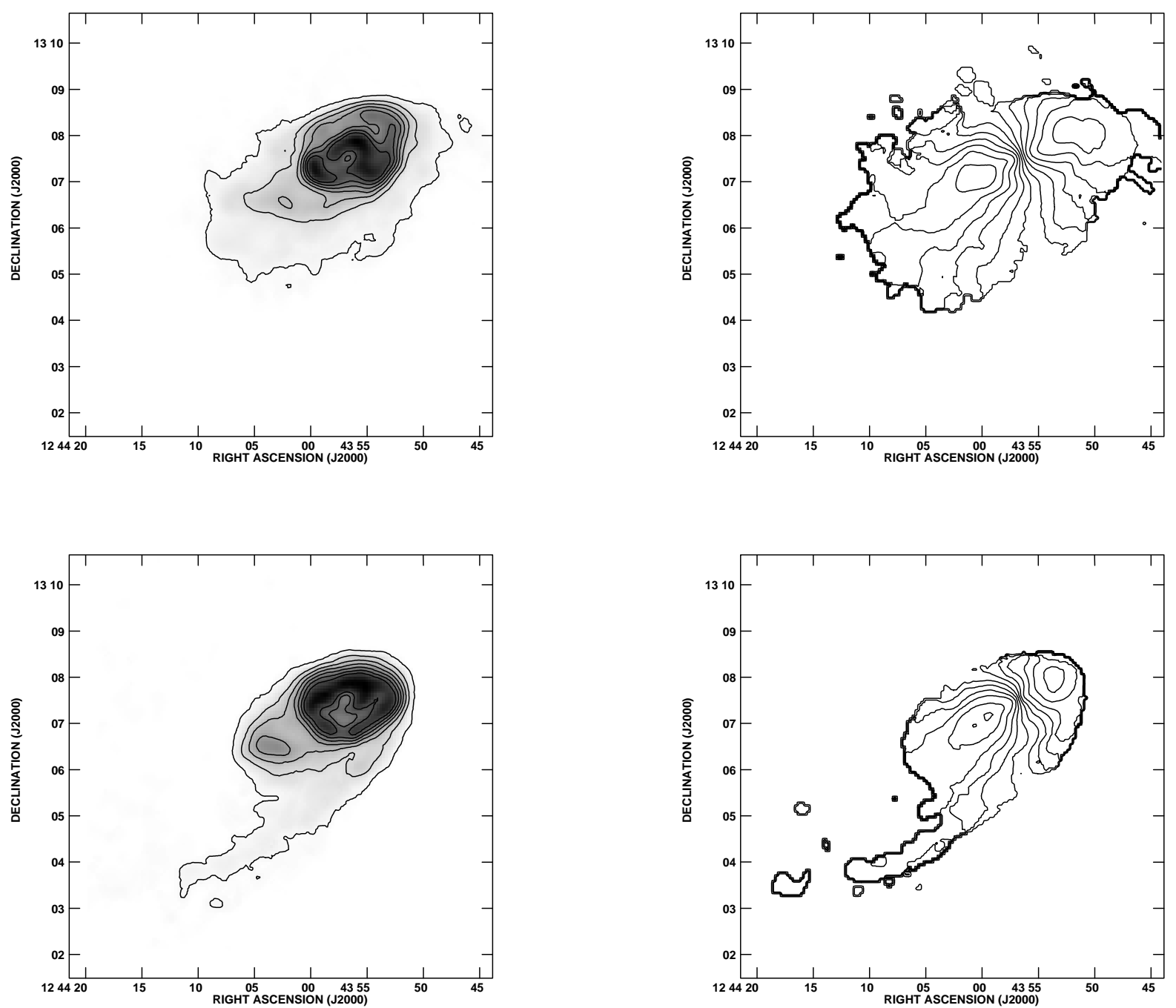

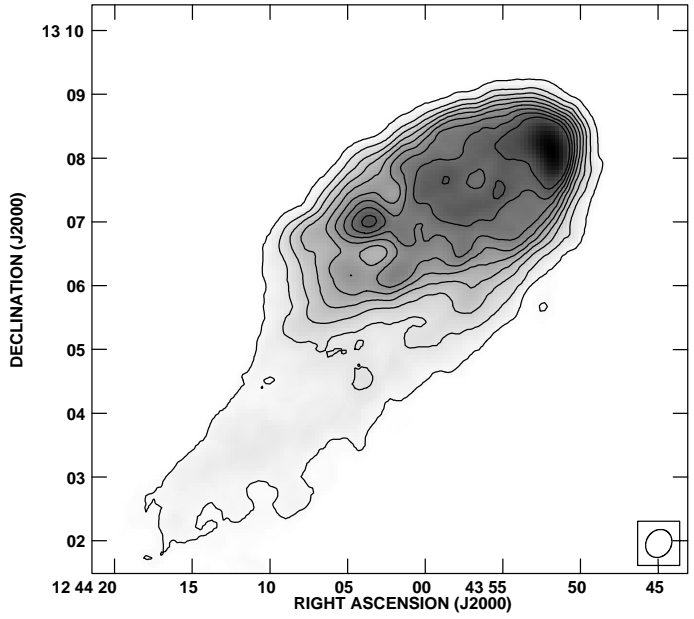

Fig. 15. Top panel: model distribution of the $\mathrm{HI}$ gas for a model of a gravitational interaction alone. The contours correspond to $(1,5$, $9,13,17,21,25) \times 10^{19} \mathrm{~cm}^{-2}$. Middle panel: distribution of the HI gas of the model of a gravitational interaction together with constant ram pressure. Bottom panel: Hi distribution of NGC 4654 (Phookun \& Mundy 1995) with the same contour levels. 

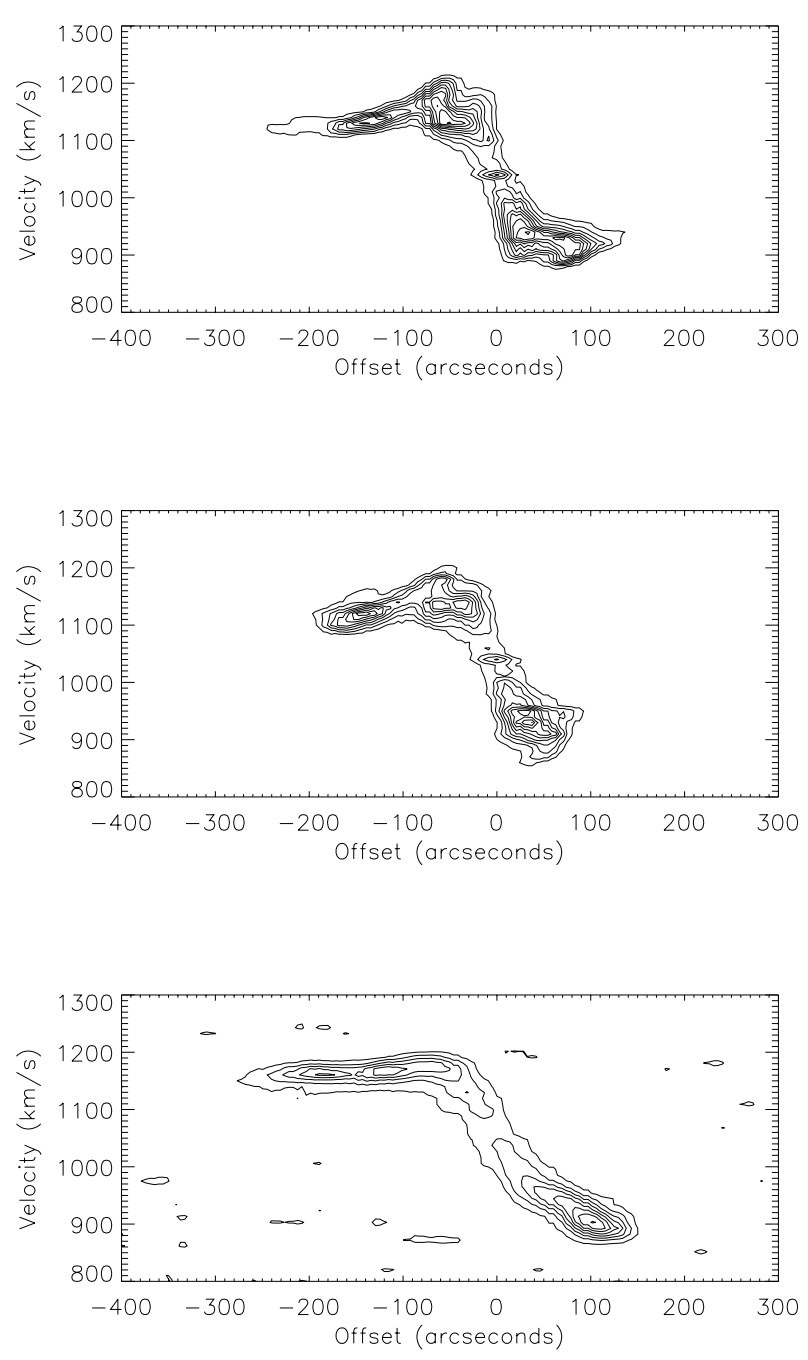

Fig. 17. Position-velocity cut through the Hi cube along the major axis. Top panel: model of a gravitational interaction alone. Middle panel: model of a gravitational interaction together with constant ram pressure. Bottom: Hi (Phookun \& Mundy 1995).

\subsection{Results}

The following results were obtained from the different models:

1. The model that uses ram pressure as the only perturbation shows a tail up to $800 \mathrm{Myr}$ after a strong ram pressure event, i.e. after the galaxy's passage through the cluster center.

2. During the ICM-ISM interaction the non-collisional component of the galaxy is heated resulting in a tail of low surface density. In the case of a fixed gravitational potential the tail has a much higher surface density, because of the lower scale height of the disk's gravitational potential.

3. An edge-on ICM-ISM interaction can produce an asymmetric stellar distribution and thus an asymmetric rotation curve.

4. An edge-on ICM-ISM interaction cannot produce bar.

5. The model using a gravitational interaction as the only perturbation can reproduce the observed asymmetry of the stellar content of NGC 4654.
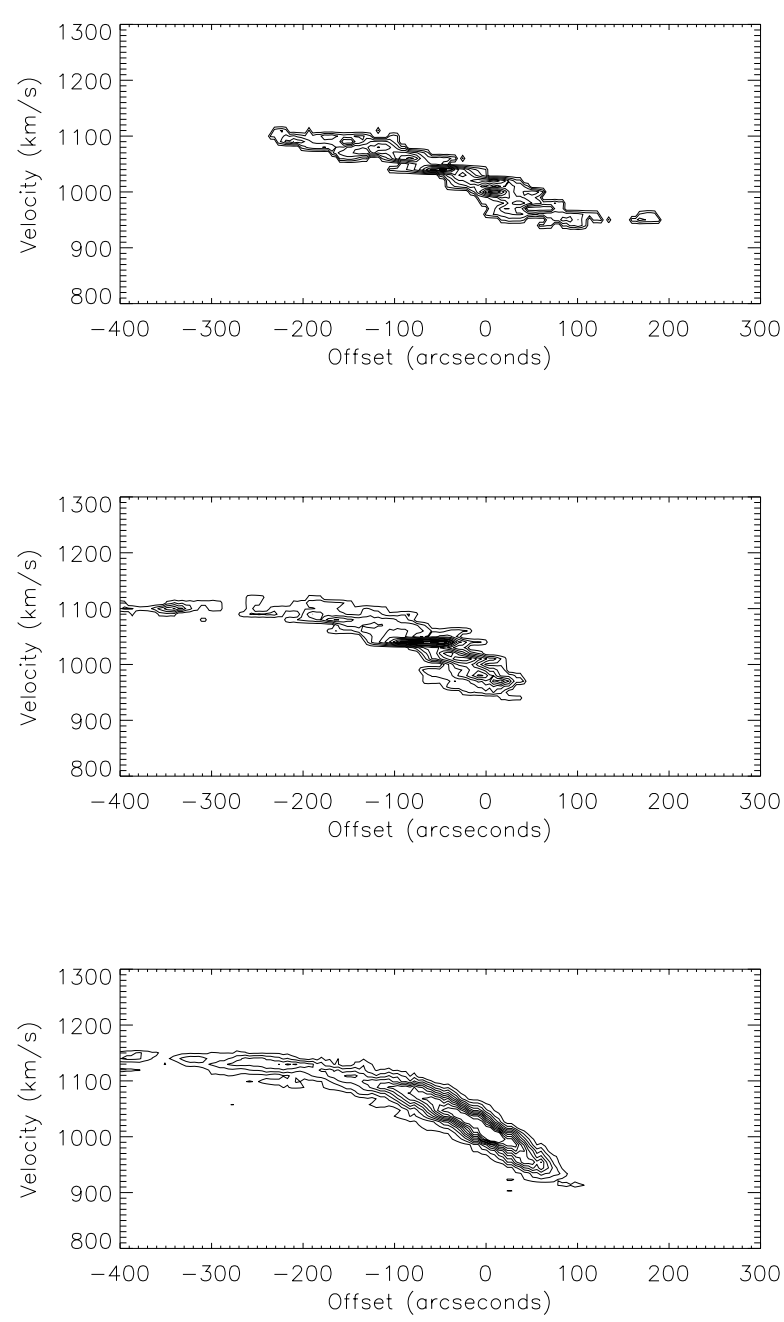

Fig. 18. Position-velocity cut through the Hi cube along the extended tail. Top panel: model of a gravitational interaction alone. Middle panel: model of a gravitational interaction together with constant ram pressure. Bottom: Hi (Phookun \& Mundy 1995).

6. The model using a gravitational interaction as the only perturbation cannot reproduce the observed, extended gas tail of NGC 4654.

7. Only the model of a mixed perturbation (gravitational and ram pressure) can account for all observed properties (gas and stars) of NGC 4654. In the case of a past tidal interaction only a small amount of ram pressure is needed to form the observed asymmetries of the gas distribution and velocity field.

8. Only the comparison with the gas distribution and velocity field can discriminate between the models.

I thus conclude that NGC 4654 has suffered most probably a tidal interaction with its companion NGC $4639 \sim 500$ Myr ago. It is now entering the cluster with a velocity of $\sim 1000 \mathrm{~km} \mathrm{~s}^{-1}$ mainly to the west. It is now experiencing a small ram pressure of the order $p_{\text {ram }} \sim 200 \mathrm{~cm}^{-3} \mathrm{~km} \mathrm{~s}^{-1}$ that is responsible for the observed extended, low surface density gas tail.

Acknowledgements. The author would like to thank L. Hernquist for making his program that generates initial conditions available, 

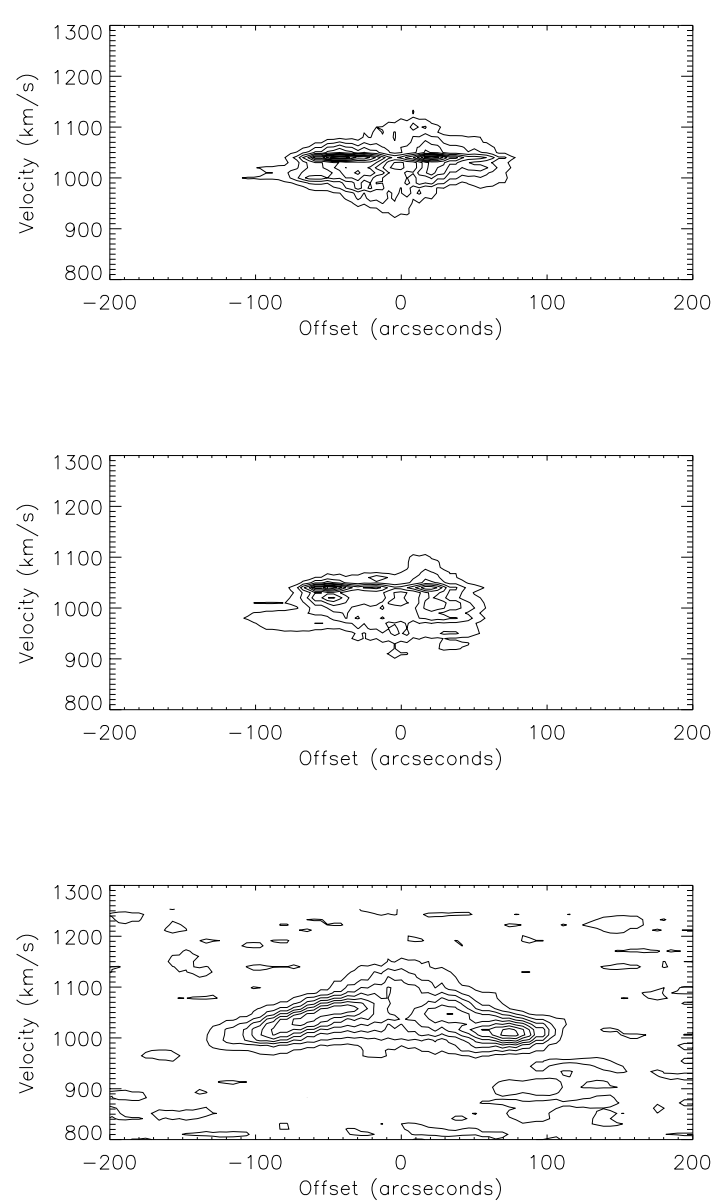

Fig. 19. Position-velocity cut through the $\mathrm{HI}_{\mathrm{r}}$ cube along the minor axis. Top panel: model of a gravitational interaction alone. Middle panel: model of a gravitational interaction together with constant ram pressure. Bottom: Hi (Phookun \& Mundy 1995).

C. Balkowski for fruitful discussions, E.M. Berkhuijsen for reading the article, and the NCSA Astronomy Digital Image Library (ADIL) for providing the data cube for this article. A special thanks to the referee, J. van Gorkom, who helped me to improve this article substantially.

\section{References}

Allen, C., \& Santillán, A. 1991, RMAA, 22, 255

Byrd, G., Freeman, T., \& Howard, S. 1993, AJ, 105, 477

Boselli, A., Tuffs, R. J., Gavazzi, G., Hippelein, H., \& Pierini, D. 1997, A\&AS, 121, 507

Cayatte, V., van Gorkom, J. H., Balkowski, C., \& Kotanyi, C. 1990, AJ, 100, 604

Cayatte, V., Kotanyi, C., Balkowski, C., \& van Gorkom, J. H. 1994, AJ, 107, 1003

de Vaucouleurs, G., de Vaucouleurs, A., Corwin, H. G., et al. 1991, Third Reference Catalogue of Bright Galaxies (New York: Springer) (RC3)

Guharthakurta, P., van Gorkom, J. H., Kotanyi, C. G., \& Balkowski, C. 1988, AJ, 96, 851

Gunn, J. E., \& Gott, J. R. 1972, ApJ, 176, 1

Hernquist, L. 1993, ApJS, 86, 389
Huchtmeier, W. K., \& Richter, O.-G. 1989, A General Catalogue of Hi observations of Galaxies (New York: Springer-Verlag)

Kenney, J. D., \& Young, J. S. 1989, ApJ, 344, 171

Phookun, B., \& Mundy, L. G. 1995, ApJ, 453, 154

Sperandio, M., Chincarini, G., Rampazzo, R., \& de Souza, R. 1995, A\&AS, 110, 279

Springel, V., Yoshida, N., \& White, D. M. 2001, NA, 6, 79

Thomasson, M., Donner, K. J., Sundelius, B., et al. 1989, A\&A, 211, 25

Vollmer, B., Cayatte, V., Balkowski, C., \& Duschl, W. J. 2001, ApJ, 561,708

Warmels, R. H. 1988 A\&AS, 72, 57

Wiegel, W. 1994, Diploma Thesis, University of Heidelberg 